\title{
Equações Integrais de Volterra em Escalas Temporais
}

\author{
Iguer L. D. Santos, \\ Departamento de Matemática, FEIS, UNESP, \\ 15385-000, Ilha Solteira, SP \\ E-mail: iguerluis@yahoo.com.br.
}

Resumo: Nesse artigo consideramos duas classes de equações integrais de Volterra em escalas temporais. Obtemos para essas classes de equações integrais, propriedades de continuidade $e$ convergência de soluções.

Palavras-chave: Equações Integrais de Volterra, Escalas Temporais, Dependência Contínua

\section{Introdução}

O cálculo em escalas temporais foi introduzido por Hilger [11] para unificar o cálculo de diferença e o cálculo diferencial. A teoria de escalas temporais admite diversas aplicações em modelagem matemática e outras áreas, conforme verificamos em [2], [4], [5], [6], [9], [12], [17] e [18]. Em particular, equações integrais de Volterra em escalas temporais foram consideradas, por exemplo, em [1], [13] e [14].

Nesse trabalho provamos propriedades de continuidade e convergência de soluções para duas classes de equações integrais de Volterra em escalas temporais. Tais propriedades constituem extensões dos respectivos casos clássicos considerados em [7] para escalas temporais.

\section{Preliminares}

Nesta seção consideramos conceitos e resultados básicos da teoria de escalas temporais que serão úteis no desenvolvimento desse trabalho.

Utilizaremos as seguintes convenções:

i) se $x \in \mathbb{R}^{n}$ denotamos a norma euclidiana de $x$ por $\|x\|$.

ii) se $A$ e $\mathbb{T}$ são subconjuntos de $\mathbb{R}$, denotamos por $A_{\mathbb{T}}$ o conjunto $A \cap \mathbb{T}$.

\subsection{Escala temporal}

Uma escala temporal $\mathbb{T} \subset \mathbb{R}$ é um conjunto não-vazio e fechado. Usaremos uma escala temporal $\mathbb{T}$ compacta, sendo $a=\min \mathbb{T}$ e $b=\max \mathbb{T}$.

Define-se a função $\sigma: \mathbb{T} \rightarrow \mathbb{T}$ como

$$
\sigma(t)=\inf \{s \in \mathbb{T}: s>t\} .
$$

Estamos supondo que $\inf \emptyset=\sup \mathbb{T}$.

Lema 2.1 ([8]) Existem $I \subset \mathbb{N} e\left\{t_{i}\right\}_{i \in I} \subset \mathbb{T}$ tal que

$$
R S:=\{t \in \mathbb{T}: t<\sigma(t)\}=\left\{t_{i}\right\}_{i \in I} .
$$




\subsection{Integração em escalas temporais}

A obtenção da $\sigma$-álgebra de subconjuntos da escala temporal $\mathbb{T}$, constituída de conjuntos $\Delta$ mensuráveis, pode ser encontrada em [10]. Denotaremos essa $\sigma$-álgebra por $\Delta$.

Para funções $f: \mathbb{T} \rightarrow \overline{\mathbb{R}}$ a noção de integração pode ser encontrada, por exemplo, em [3], [15] e [16]. Denotamos a integral de uma função $f: \mathbb{T} \rightarrow \overline{\mathbb{R}}$ sobre um conjunto $E \in \Delta$ por

$$
\int_{E} f(s) \Delta s
$$

Chamamos essa integral de $\Delta$-integral de Lebesgue de $f$ sobre $E$. Denotaremos por $L_{1}\left(E, \mathbb{R}^{n}\right)$ o conjunto das funções $f: \mathbb{T} \rightarrow \mathbb{R}^{n} \Delta$-integráveis sobre $E$.

Dada uma função $f: \mathbb{T} \rightarrow \mathbb{R}^{n}$ defina $\tilde{f}:[a, b] \rightarrow \mathbb{R}^{n}$ por

$$
\tilde{f}(t)=\left\{\begin{array}{l}
f(t), t \in \mathbb{T} \\
f\left(t_{i}\right), \quad t \in\left(t_{i}, \sigma\left(t_{i}\right)\right) \text { para algum } i \in I,
\end{array}\right.
$$

onde $I \subset \mathbb{N}$ e $\left\{t_{i}\right\}_{i \in I}=R S$.

Seja $E \subset \mathbb{T}$ e defina

$$
\tilde{E}=E \cup \bigcup_{i \in I_{E}}\left(t_{i}, \sigma\left(t_{i}\right)\right)
$$

onde

$$
I_{E}:=\left\{i \in I: t_{i} \in E \cap R S\right\} .
$$

O teorema a seguir é provado em [8] para funções escalares. Entretanto, esse resultado continua válido para funções vetoriais, como afirmado a seguir.

Teorema 2.1 ([8]) Seja $E \in \Delta$ tal que $b \notin E$. Então, $f \in L_{1}\left(E, \mathbb{R}^{n}\right)$ se, e somente se, $\tilde{f} \in L_{1}\left(\tilde{E}, \mathbb{R}^{n}\right)$. Neste caso,

$$
\int_{E} f(s) \Delta s=\int_{\tilde{E}} \tilde{f}(s) d s .
$$

Usaremos também a seguinte generalização do Lema de Gronwall.

Teorema 2.2 Sejam $\varphi:\left[t_{0}, t_{1}\right] \rightarrow[0,+\infty)$ uma função Lebesgue integrável e $\psi:\left[t_{0}, t_{1}\right] \rightarrow$ $[0,+\infty)$ uma função contínua. Suponha que

$$
\varphi(t) \leq K+L \int_{t_{0}}^{t} \psi(s) \varphi(s) d s
$$

para todo $t \in\left[t_{0}, t_{1}\right]$, com $K \geq 0$ e $L \geq 0$. Então

$$
\varphi(t) \leq K \exp L \int_{t_{0}}^{t} \psi(s) d s
$$

para todo $t \in\left[t_{0}, t_{1}\right]$.

\section{Equações integrais de Volterra}

Sejam $g: \mathbb{T} \times \mathbb{T} \times \mathbb{R}^{n} \rightarrow \mathbb{R}^{n}$ e $f: \mathbb{T} \rightarrow \mathbb{R}^{n}$ funções dadas. Nesse trabalho consideramos as seguintes equações integrais de Volterra em escalas temporais

$$
\begin{gathered}
x(t)=f(t)+\int_{[a, t)_{\mathbb{T}}} g(t, s, x(s)) \Delta s \\
x(t)=f(t)+\int_{[a, t)_{\mathbb{T}}} g(t, s, x(\sigma(s))) \Delta s
\end{gathered}
$$

onde $t \in \mathbb{T}$ e $x: \mathbb{T} \rightarrow \mathbb{R}^{n}$ é a função incógnita.

A existência de soluções contínuas para essas duas classes de equações integrais podem ser encontradas em [13]. 


\section{Continuidade e convergência de soluções}

Nessa seção obtemos as extensões das propriedades de continuidade e convergência de soluções consideradas em [7] para escalas temporais.

\subsection{Continuidade de soluções}

Consideramos primeiro a continuidade de soluções com relação às condições iniciais para a equação 1. Outro resultado de dependência contínua para essa classe de equações integrais foi obtido em [13]. Assim, sejam $x(t)$ e $y(t)$ soluções das equações

$$
x(t)=f_{1}(t)+\int_{[a, t)_{\mathbb{T}}} g(t, s, x(s)) \Delta s
$$

$\mathrm{e}$

$$
y(t)=f_{2}(t)+\int_{[a, t)_{\mathbb{T}}} g(t, s, y(s)) \Delta s
$$

em $\mathbb{T}$, com $\left\|f_{1}-f_{2}\right\|_{\infty} \leq \delta$, então para $g$ de Lipschitz devemos ter $\|x-y\|_{\infty} \leq \epsilon$.

Teorema 4.1 Sejam $f_{1}, f_{2}: \mathbb{T} \rightarrow \mathbb{R}^{n}$ e $g: U \rightarrow \mathbb{R}^{n}$ funções contínuas, com

$$
U=\left\{(t, s, x): a \leq s \leq t \leq b, x \in \mathbb{R}^{n}\right\} .
$$

Suponha que exista $L>0$ tal que $|g(t, s, x)-g(t, s, y)| \leq L|x-y|$ em $U$. Sejam $x(t)$ e $y(t)$ soluções de 3 e 4 , respectivamente, em $\mathbb{T}$. Se $\delta=\left\|f_{1}-f_{2}\right\|_{\infty}$ então

$$
\|x(t)-y(t)\| \leq \delta e^{L(t-a)}
$$

para todo $t \in \mathbb{T}$.

A demonstração desse teorema segue nas mesmas linhas da demonstração do respectivo caso contínuo [7]. Para isso são usados os teoremas 2.1 e 2.2.

Consideramos agora a continuidade de soluções com relação às condições iniciais para a equação 2. Assim, sejam $x(t)$ e $y(t)$ soluções das equações

$$
x(t)=f_{1}(t)+\int_{[a, t)_{\mathbb{T}}} g(t, s, x(\sigma(s))) \Delta s
$$

$\mathrm{e}$

$$
y(t)=f_{2}(t)+\int_{[a, t)_{\mathbb{T}}} g(t, s, y(\sigma(s))) \Delta s
$$

em $\mathbb{T}$.

Teorema 4.2 Sejam $f_{1}, f_{2}: \mathbb{T} \rightarrow \mathbb{R}^{n}$ e $g: U \rightarrow \mathbb{R}^{n}$ funções contínuas, com

$$
U=\left\{(t, s, x): a \leq s \leq t \leq b, x \in \mathbb{R}^{n}\right\} .
$$

Suponha que exista $L>0$ tal que $|g(t, s, x)-g(t, s, y)| \leq L|x-y|$ em $U$. Sejam $x(t)$ e $y(t)$ soluções de 5 e 6 , respectivamente, em $\mathbb{T}$. Se $\delta=\left\|f_{1}-f_{2}\right\|_{\infty}$ e $L(b-a)<1$ então

$$
\|x(t)-y(t)\| \leq \delta+M \delta e^{M L(t-a)}
$$

para todo $t \in \mathbb{T}$, onde $M=\frac{1}{1-(b-a) L}$. 


\subsection{Convergência de soluções}

Obtemos a seguir resultados de convergência de soluções para equações integrais de Volterra em escalas temporais.

Teorema 4.3 Seja $\left\{g_{k}\right\}$ uma sequência de funções contínuas, com $g_{k}: \mathbb{T} \times \mathbb{T} \times \mathbb{R}^{n} \rightarrow \mathbb{R}^{n}$ satisfazendo

$$
\left\|g_{k}(t, s, x)\right\| \leq C(1+\|x\|)
$$

para todo $(t, s, x) \in \mathbb{T} \times \mathbb{T} \times \mathbb{R}^{n}$. Suponha que $\left\{f_{k}\right\}$ é uma sequência de funções $f_{k}: \mathbb{T} \rightarrow \mathbb{R}^{n}$ uniformemente limitada e equicontínua tal que $f_{k} \rightrightarrows f$. Suponha também que

(a) $g_{k}(t, s, x) \rightarrow g(t, s, x) e m \mathbb{T} \times \mathbb{T} \times \mathbb{R}^{n}$.

(b) existe $L>0$ tal que $\left\|g_{k}(t, s, x)-g_{k}(t, s, y)\right\| \leq L\|x-y\|$ para todo $k$.

(c) para cada $k, \psi_{k}(t)$ é uma solução contínua de

$$
\psi_{k}(t)=f_{k}(t)+\int_{[a, t)_{\mathbb{T}}} g_{k}\left(t, s, \psi_{k}(s)\right) \Delta s
$$

$t \in \mathbb{T}$.

(d) para cada $\epsilon>0$ e $M>0$, existe $\delta>0$ tal que

$$
\left\|g_{k}(t, s, x)-g_{k}\left(t_{1}, s, x\right)\right\| \leq \epsilon\left|t-t_{1}\right|
$$

onde $s \in \mathbb{T},\left|t-t_{1}\right|<\delta, t, t_{1} \in \mathbb{T},\|x\| \leq M$ e $k$ é um inteiro.

Então existe uma subsequência $\left\{\psi_{k_{j}}\right\} \subset\left\{\psi_{k}\right\}$ e uma função $\psi: \mathbb{T} \rightarrow \mathbb{R}^{n}$ tal que $\psi_{k_{j}} \rightrightarrows \psi$, e $\psi$ satisfaz

$$
\psi(t)=f(t)+\int_{[a, t)_{\mathbb{T}}} g(t, s, \psi(s)) \Delta s
$$

$e m \mathbb{T}$.

A demonstração desse teorema também segue nas mesmas linhas da demonstração do respectivo caso contínuo [7]. Além disso, são usados os teoremas 2.1 e 2.2 na obtenção desse resultado.

Um resultado de convergência análogo também pode ser obtido para a classe de equações 2 .

Teorema 4.4 Seja $\left\{g_{k}\right\}$ uma sequência de funções contínuas, com $g_{k}: \mathbb{T} \times \mathbb{T} \times \mathbb{R}^{n} \rightarrow \mathbb{R}^{n}$ satisfazendo

$$
\left\|g_{k}(t, s, x)\right\| \leq C(1+\|x\|)
$$

para todo $(t, s, x) \in \mathbb{T} \times \mathbb{T} \times \mathbb{R}^{n}$. Suponha que $\left\{f_{k}\right\}$ é uma sequência de funções $f_{k}: \mathbb{T} \rightarrow \mathbb{R}^{n}$ uniformemente limitada e equicontínua tal que $f_{k} \rightrightarrows f$. Suponha também que

(a) $g_{k}(t, s, x) \rightarrow g(t, s, x)$ em $\mathbb{T} \times \mathbb{T} \times \mathbb{R}^{n}$.

(b) $\left\|g_{k}(t, s, x)-g_{k}(t, s, y)\right\| \leq L\|x-y\|$ para todo $k$.

(c) para cada $k, \psi_{k}(t)$ é uma solução contínua de

$$
\psi_{k}(t)=f_{k}(t)+\int_{[a, t)_{\mathbb{T}}} g_{k}\left(t, s, \psi_{k}(\sigma(s))\right) \Delta s,
$$

$t \in \mathbb{T}$. 
(d) para cada $\epsilon>0$ e $M>0$, existe $\delta>0$ tal que

$$
\left\|g_{k}(t, s, x)-g_{k}\left(t_{1}, s, x\right)\right\| \leq \epsilon\left|t-t_{1}\right|
$$

quando $s \in \mathbb{T},\left|t-t_{1}\right|<\delta, t, t_{1} \in \mathbb{T},\|x\| \leq M$ e $k$ é um inteiro.

Se $C(b-a)<1$, então existe uma subsequência $\left\{\psi_{k_{j}}\right\} \subset\left\{\psi_{k}\right\}$ e uma função $\psi: \mathbb{T} \rightarrow \mathbb{R}^{n}$ tal que $\psi_{k_{j}} \rightrightarrows \psi$, e $\psi$ satisfaz

$$
\psi(t)=f(t)+\int_{[a, t)_{\mathbb{T}}} g(t, s, \psi(\sigma(s))) \Delta s
$$

$e m \mathbb{T}$.

\section{Conclusões}

Esse trabalho contribuiu para o avanço do conhecimento na teoria de escalas temporais. Mais especificamente, usando uma generalização do Lema de Gronwall prova propriedades de continuidade e convergência de soluções contínuas para equações integrais de Volterra em escalas temporais.

\section{Referências}

[1] M. Adivar and Y. N. Raffoul. Existence of resolvent for Volterra integral equations on time scales. Bull. Aust. Math. Soc., 82(1):139-155, 2010.

[2] R. Agarwal, M. Bohner, D. O'Regan, and A. Peterson. Dynamic equations on time scales: a survey. Journal of Computational and Applied Mathematics, 141(1):1-26, 2002.

[3] R. G. Bartle. The elements of integration and Lebesgue measure. John Wiley \& Sons Inc., New York, 1995.

[4] M. Bohner and G. S. Guseinov. Surface areas and surface integrals on time scales. Dynam. Systems Appl., 19(3-4):435-453, 2010.

[5] M. Bohner, G. S. Guseinov, and B. Karpuz. Properties of the Laplace transform on time scales with arbitrary graininess. Integral Transforms Spec. Funct., 22(11):785-800, 2011.

[6] M. Bohner and A. Peterson. Advances in dynamic equations on time scales. Birkhäuser Boston Inc., Boston, MA, 2003.

[7] T. A. Burton. Volterra integral and differential equations, volume 202 of Mathematics in Science and Engineering. Elsevier B. V., Amsterdam, second edition, 2005.

[8] A. Cabada and D. R. Vivero. Expression of the lebesgue $\delta$-integral on time scales as a usual lebesgue integral; application to the calculus of $\delta$-antiderivatives. Mathematical and Computer Modelling, 43(1):194-207, 2006.

[9] M. Federson, J. G. Mesquita, and A. Slavík. Measure functional differential equations and functional dynamic equations on time scales. Journal of Differential Equations, $252(6): 3816-3847,2012$.

[10] G. S. Guseinov. Integration on time scales. Journal of Mathematical Analysis and Applications, 285(1):107-127, 2003.

[11] S. Hilger. Analysis on measure chains - a unified approach to continuous and discrete calculus. Results Math., 18(1-2):18-56, 1990. 
[12] R. Hilscher and V. Zeidan. First order conditions for generalized variational problems over time scales. Computers \& Mathematics with Applications, 62(9):3490-3503, 2011.

[13] T. Kulik and C. C. Tisdell. Volterra integral equations on time scales: basic qualitative and quantitative results with applications to initial value problems on unbounded domains. Int. J. Difference Equ., 3(1):103-133, 2008.

[14] D. B. Pachpatte. On a nonstandard Volterra type dynamic integral equation on time scales. Electron. J. Qual. Theory Differ. Equ., (72):1-14, 2009.

[15] H. L. Royden. Real analysis. Macmillan Publishing Company, New York, third edition, 1988.

[16] W. Rudin. Real and complex analysis. McGraw-Hill Book Co., New York, third edition, 1987.

[17] I. L. D. Santos and G. N. Silva. Absolute continuity and existence of solutions to dynamic inclusions in time scales. Mathematische Annalen, 356:373-399, 2013.

[18] Z. Zhan, W. Wei, and H. Xu. Hamilton-jacobi-bellman equations on time scales. Mathematical and Computer Modelling, 49(9-10):2019-2028, 2009. 